\title{
Reported falls in a tertiary care psychiatry hospital in year 2018- A preliminary retrospective review
}

\author{
Akuratiyage L.Y. ${ }^{[1]}$ Sheriff R. ${ }^{[2]}$ Amarasinghe S.C. ${ }^{[3]}$
}

\begin{abstract}
1) Registrar in psychiatry, National Institute of Mental Health, Sri Lanka..
2) Medical officer, Planning unit, National Institute of Mental Health, Sri Lanka.

3) Consultant Psychiatrist, National Institute of Mental Health, Sri Lanka.
\end{abstract}

\section{Background}

Falls in psychiatry units are generally commoner than other age-matched units. Falls can be potentially serious events and can result in substantial morbidity and mortality. It can adversely affect patient outcomes and cause a burden to the staff. A range of causes has been identified to contribute to falls in psychiatry units. Proper surveillance systems are capable of proposing steps to mitigate these falls.

\section{Objectives}

-To explore the existing surveillance system on falls during ward stay in National Institute of Mental Health (NIMH)

- To explore the reported falls during 2018 in NIMH

\section{Method}

The study group went through total 325 hospital incident forms filled by nursing officers during inpatient falls during 2018. And we retrospectively analyzed these secondary data to describe patterns of falls.

\section{Results}

Out of reported falls,

$>181(55 \%)$ were males.

$>$ Out of diagnoses of fallen individuals,

Schizophrenia (34\%),

Bipolar Affective Disorder (22\%) and

Dementia (15\%) are the commonest.

$>$ Falls are commonly noted during 6am - 8am (20\%) and 10am-12noon (15\%).

$>$ Commonest age groups are 60-69yrs (25\%) and $50-59$ yrs (22\%).

$>$ These have mainly occurred within the ward $(86 \%)$ and inside the bathroom (23\%) due to wet floor.
Five fractures were noted in above 50 age group without reported mortality.

\section{$24(7 \%)$ were due to convulsions.}

$>$ The sections of the form describing causes and preceding events to fall were filled poorly in comparison to other sections.

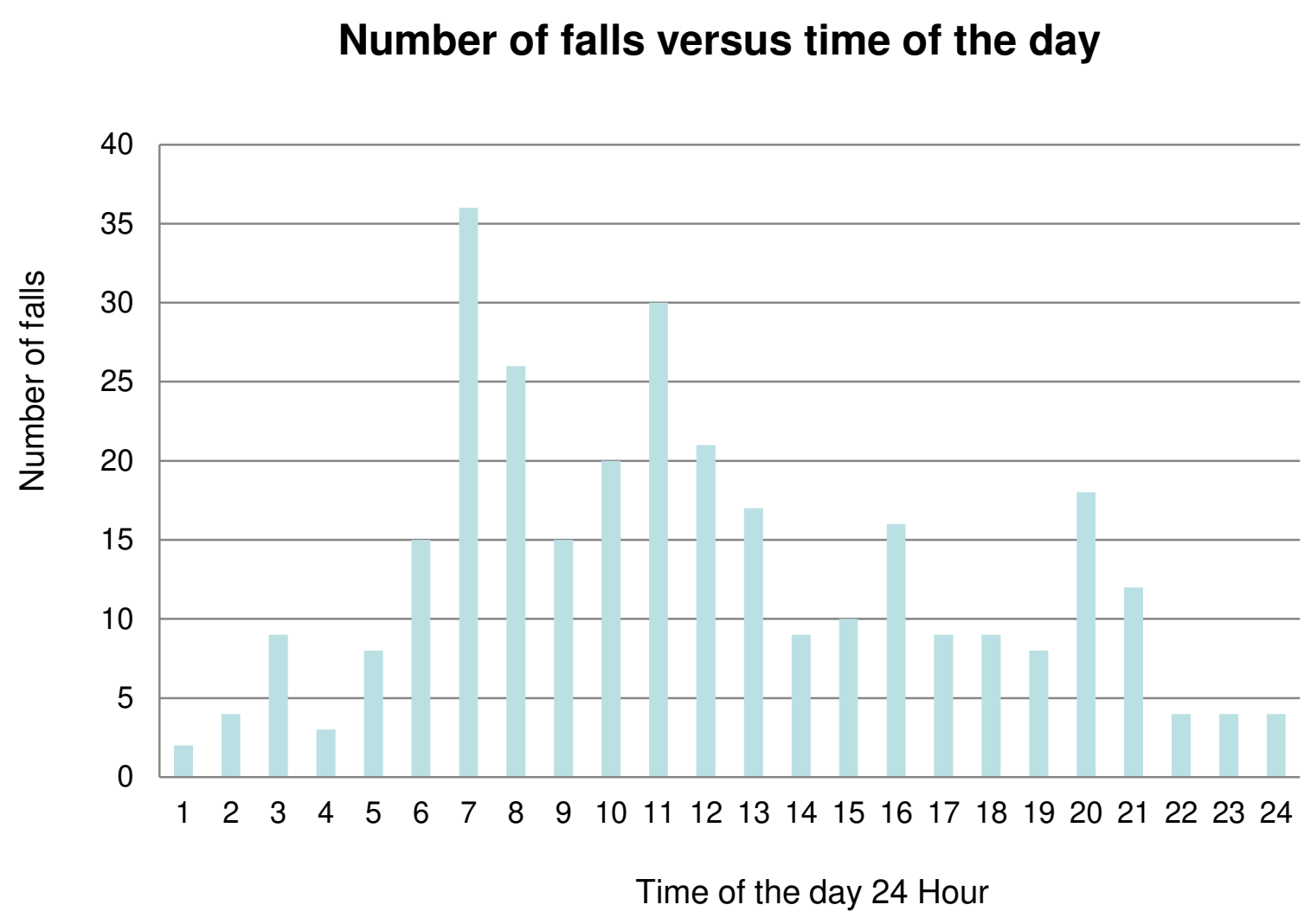

\section{Conclusions}

Falls are commoner among males and in over 50 age groups.

$>$ They are more frequent during morning.

$>$ Modifiable factors such as wet floors might need intervention.

$>$ The incident form does not give satisfactory insight in to the cause (Particularly effect of medications).

$>$ The identified patterns need to be complemented with total admissions and length of stays to arrive in to conclusive causalities.

$>$ Incident forms need modifications to have better clinical utility. 